\title{
Pleiotropy of Presenilins
}

\section{Airong Li* and Rudolph E Tanzi}

Genetics and Aging Research Unit, Department of Neurology, Mass General Institute for Neurodegenerative Diseases, Massachusetts General Hospital and Harvard Medical School, MA, USA

\begin{abstract}
Pleiotropy genes affect multiple and apparently unrelated phenotypes. Here we describe pleiotropy gene presenilins, mutations in which have been detected in three genetically heterogeneous diseases: early-onset familial Alzheimer's disease, familial or sporadic dilated cardiomyopathy, and familial hidradenitis suppurativa.
\end{abstract}

Pleiotropy describes the genetic effect of a single gene on multiple phenotypic traits, or genetic variations in a single gene lead to multiple unrelated traits or diseases [1]. In contrast to pleiotropy, genetic heterogeneity means that a single phenotype or genetic disorder may be caused by different alleles in the same gene (allelic heterogeneity), or variations in two or more unrelated gene loci (locus heterogeneity) [2]. Pleiotropy genes affect multiple and apparently unrelated phenotypes [3], such as mutations in the cystic fibrosis transmembrane conductance regulator (CFTR) gene led to either cystic fibrosis [4] or congenital bilateral absence of vas deferens [5]; mutations in the phenylalanine hydroxylase $(\mathrm{PAH})$ gene caused not only phenylketonuria (PKU) but also a number of phenotypes, including mental retardation, eczema, and pigment defects [6]. The underlying mechanism of pleiotropy genes may include that their encoded gene products either are used by various cells or have different functional targets. For example, loss-of-function mutations in the RET protooncogene (RET) gene led to Hirschsprung disease (HSCR) by impairing its tyrosine kinase activity (i.e. R972G), disrupting the correct maturation of the RET protein and its transport to the cell surface (i.e. S32L and F393L) $[7,8]$, or loss of function effect in homozygous condition (i.e. C620R/C620R) [9]. In contrast, two gain-of-function mutations in the RET, heterozygous C620R resulting in a dominant gain of function effect with low penetrance [9], and the Y606C increasing receptor dimerization and constitutively activating receptor, were associated with multiple endocrine neoplasia type $2 \mathrm{~A}$ (MEN2A) [9-11].

Presenilins were first identified in screens for mutations causing early onset forms of familial Alzheimer's disease (AD) in 1995 [12-14]. Of two presenilin genes in human, PSEN1 is located on chromosome 14 that encodes presenilin 1 (PS1), and PSEN2 is on chromosome 1 that codes for presenilin 2 (PS2). PS1 and PS2 are highly conserved polytopic membrane proteins that form the catalytic centre of the $\gamma$-secretase complex [15]. The $\gamma$-secretase consists of a large protein complex that include at least four proteins, presenilins (PS1, PS2), nicastrin (NCSTN), anterior pharynx defective 1 (APH1) and presenilin enhancer 2 (PEN2), and is responsible for the proteolytic processing of a variety of membrane-associated fragments derived from type I integral membrane proteins, including the amyloid precursor protein (APP) and the Notch [16]. Notch receptor cleavage within the plasma membrane by $\gamma$-secretase results in the release and translocation of the Notch intracellular domain (NICD) into the nucleus to execute Notch signaling, which is critical for development. Presenilin mutations have been detected in three unrelated and genetically heterogeneous diseases: early-onset familial AD [15], familial or sporadic dilated cardiomyopathy (DCM) $[17,18]$, and familial hidradenitis suppurativa (HS) [19].

\section{Presenilin and AD}

$\mathrm{AD}$ is a progressive neurodegenerative disease and the most common form of dementia in the elderly, affecting one in 10 individuals over 65 and nearly half over 85 years old (http://www.alz.org/) [20] $\mathrm{AD}$ is characterized clinically by cognitive decline accompanied by neuronal loss and the formation of neurofibrillary tangles (NFTs) and insoluble toxic amyloid plaques in the brain [21]. Genetically, AD is heterogeneous and complex [22]. The majority of $\mathrm{AD}$ patients have the late-onset form ( $>60$ years). Roughly $5-10 \% \mathrm{AD}$ occurs in families where the disease is autosomal dominantly inherited with full penetrance and early onset ( $<60$ years). Presenilin mutations underlie the majority of cases of early-onset familial AD [15]. To date, approximately 167 mutations in PSEN1 and 14 mutations in PSEN2 have been identified in familial $\mathrm{AD}$ patients (http://www. alz.org/; http://www.molgen. ua.ac.be/ADMutations). Majority of mutations in PSEN1 and PSEN2 are missense mutations that result in single amino-acid substitutions, and other type of mutations such as in-frame deletions, insertions or splice site mutations are also found (http://www.molgen.ua.ac.be/ ADMutations). PSEN2-K115E fx10 was the only frame-shift mutation detected in presenilin coding region in $\mathrm{AD}$ patients [23].

Mutations in presenilins alter the proteolytic processing of APP by aberrant $\gamma$-secretase activities that results in increased neurotoxic $\beta$-amyloid (A $\beta$ ) 42 production [21]. Moreover, PSEN1 mutations may perturb cellular $\mathrm{Ca}^{2+}$ homeostasis thereby render neurons more vulnerable to excitotoxity and apoptosis to promote neuronal degeneration [24-27]. Transgenic mice overexpressing the early onset familial AD-associated APP and PSEN1 mutations resulted in accelerated $A \beta$ accumulation and associative learning deficits [28]. A transgenic conditional double knockout mouse lacking both PSEN1 and PSEN2 in the postnatal forebrain showed impaired hippocampal memory and synaptic plasticity and developed neurodegeneration of the cerebral cortex [29]. These findings indicate that loss of function in presenilins plays an important role in $\mathrm{AD}$ pathogenesis.

\section{Presenilin and DCM}

DCM is defined clinically by dilation and impaired contraction of one or both ventricles. DCM occurs in all ages, most common between 20-60 years. DCM causes roughly one-third of cases of congestive heart failure [30]. About $20-40 \%$ of patients have familial forms of the

*Corresponding author: Airong Li,Genetics and Aging Research Unit Massachusetts General Hospital, Harvard Medical School, CNY114, 16th Street Charlestown, MA 02129, USA, Tel: 617-724-9397; Fax: 617-724-1823; E-mail: ali3@partners.org

Received October 18, 2012; Accepted October 18, 2012; Published October 20 2012

Citation: Li A, Tanzi RE (2012) Pleiotropy of Presenilins. Hereditary Genet 2: e105. doi:10.4172/2161-1041.1000e105

Copyright: (c) $2012 \mathrm{Li} \mathrm{A}$, et al. This is an open-access article distributed under the terms of the Creative Commons Attribution License, which permits unrestricted use, distribution, and reproduction in any medium, provided the original author and source are credited. 
disease [31]. Genetically, DCM is heterogeneous [32,33]. Mutations in presenilins have been detected in all clinically affected subjects with heart failure from three DCM families [17], as well as four severe sporadic DCM patients who were performed heart transplantation due to heart failure [18].

So far six promoter region variants or missense mutations in presenilins have been identified in patients with DCM $[17,18]$. Two PSEN1 5' promoter region variants, $-92 \mathrm{delC}$ and $-21 \mathrm{G}>\mathrm{A}$, led to a significant reduction in transcriptional activities and a decreased expression of PS1 protein in the myocardium, suggesting loss of function [18]. Two PSEN1 missense mutations, PSEN1-E318G and PSEN1-D333G, were 15 amino acids apart and located in a "hot spot" exclusively in a large hydrophilic cytoplasmic loop between PS1 transmembrane domains six and seven. This region contained critical functional domains which were processed by endoproteolysis [34], and were essential for the interaction between PS1 and other proteins [35], such as binding PS1 to ubiquilin1, which was associated with an increased susceptibility risk for late-onset AD $[35,36]$. High prevalence of PSEN1 missense mutations also occurred in or around in this loop region in familial AD [37-39], consisting of $12.6 \%(21 / 167)$ of all reported PSEN1 mutations (http://www.hgmd.cf.ac.uk/). Two PSEN2 missense mutations, PSEN2-R62H and PSEN2-S130L, were located in a "hot spot" of the PS2 NH2 terminus $[17,18]$. Calcium signaling was altered in cultured skin fibroblasts from PSEN1-D333G and PSEN2S130L mutation carriers [17]. The PSEN2-R62H missense mutation enhanced PS2 degradation, reduced PS2 stability in mouse embryonic fibroblasts, and altered compromised PS2 function in Notch signaling in C. elegans [40]. Interestingly, these two "hot spots" in which missense mutations in presenilins were clustered are highly divergent regions of PS1 and PS2, as both proteins share extensive sequence identity along their entire lengths except their NH2-terminus and the second half of their hydrophilic loop regions. Presenilin missense mutations in divergent regions may pay an important role in DCM pathogenesis by modulating different functions of PS1 and PS2 [18].

PSEN1 and PSEN2 were strongly expressed in the heart and essential for cardiac morphogenesis [41,42]. Mice null for PS1 were either dead at birth $\left(\mathrm{Ps}^{-1 /}\right)$ or embryonically lethal $\left(\mathrm{Ps}^{-/ /} / \mathrm{Ps}^{-/+}\right)$, exhibiting multiple developmental defects including a cardiac anomaly [42]. Studies in transgenic Drosophila models with an emerging optical coherence tomography imaging technology demonstrated that changes in presenilin level either by overexpression or silencing of the Drosophila ortholog of presenilins led to cardiac dysfunction in adult flies, owing to aberrant calcium channel receptor activities and disrupted Wnt signaling transduction, indicating a pathogenic role for presenilin mutations in DCM pathogenesis [43].

\section{Presenilin and HS}

HS is a chronic inflammatory skin disease and severe form of acne (acne inversa). HS affects the apocrine sweat glands or sebaceous glands most commonly in areas of the underarms, breasts, inner thighs, groin and buttocks, and often results in chronically draining sinus tracks, wounds, scarred and tissue immobility [44]. The prevalence of HS is approximately 1 in 300 persons, with females affected three times more often than males. Genetics plays a significant role in the etiology of HS. Genetically, HS is heterogeneous [45].

A single PSEN1-P242LfsX11 frame-shift mutation was found to segregate with HS in a family with HS where the disease was autosomal dominantly inherited [19]. In addition, heterozygous loss-of-function mutations were found in genes encoding the other components and cofactor of $\gamma$-secretase complex: including NCSTN
(R117X, E584DfsX44, and A486_T517del) and PEN2 (F23LfsX46 and F94SfsX49) in patients with familial HS [19]. RT-PCR showed a significantly reduced PSEN1 or NCSTN transcript expression in peripheral lymphocytes of the affected individuals carrying a PSEN1 or NCSTN mutation. These findings suggest that loss of function in the $\gamma$-secretase component genes may be a genetic basis for familial HS [19]. None of 15 HS patients from HS families at 50 years or older carrying a mutation in a $\gamma$-secretase complex component had symptoms of AD [19]. Notably, HS-associated PSEN1-P242LfsX11 [19] and AD-associated PSEN2-K115E fx10 [23] were only frameshift mutations detected in presenilin coding regions that resulted in premature termination codons.

Collectively, mutations or variants in presenilins were detected in patients with $\mathrm{AD}, \mathrm{DCM}$ or HS, which indicate pleiotropy of presenilins. Functional analyses have suggested that the mutations or variants in presenilins contribute to $\mathrm{AD}, \mathrm{DCM}$ or HS pathogenesis through loss of gene function. Further studies are warranted to elucidate the molecular mechanisms involved in how mutations or variants in the same presenilin may result in distinct pathogenic features in three unrelated diseases: neurodegeneration in $\mathrm{AD}$, cardiomyopathy in $\mathrm{DCM}$, or acne in HS.

\section{References}

1. Hodgkin J (1998) Seven types of pleiotropy. Int J Dev Biol 42: 501-505.

2. McClellan J, King MC (2010) Genetic heterogeneity in human disease. Cell 141: 210-217.

3. Caspari E (1952) Pleiotropic gene action. Evolution 6: 1-18

4. Riordan JR, Rommens JM, Kerem B, Alon N, Rozmahel R, et al. (1989) Identification of the cystic fibrosis gene: cloning and characterization of complementary DNA. Science 245: 1066-1073.

5. Anguiano A, Oates RD, Amos JA, Dean M, Gerrard B, et al. (1992) Congenital bilateral absence of the vas deferens. A primarily genital form of cystic fibrosis. JAMA 267: 1794-1797.

6. Paul D (2000) A double-edged sword. Nature 405: 515.

7. Romeo G, Ronchetto P, Luo Y, Barone V, Seri M, et al. (1994) Poin mutations affecting the tyrosine kinase domain of the RET proto-oncogene in Hirschsprung's disease. Nature 367: 377-378.

8. Carlomagno F, De Vita G, Berlingieri MT, de Franciscis V, Melillo RM, et al (1996) Molecular heterogeneity of RET loss of function in Hirschsprung's disease. EMBO J 15: 2717-2725.

9. Yin L, Puliti A, Bonora E, Evangelisti C, Conti V, et al. (2007) C620R mutation of the murine ret proto-oncogene: loss of function effect in homozygotes and possible gain of function effect in heterozygotes. Int J Cancer 121: 292-300.

10. Ercolino T, Lombardi A, Becherini L, Piscitelli E, Cantini G, et al. (2008) The Y606C RET mutation causes a receptor gain of function. Clin Endocrinol (Oxf) 69: $253-258$

11. Mulligan LM, Kwok JB, Healey CS, Elsdon MJ, Eng C, et al. (1993) Germ-line mutations of the RET proto-oncogene in multiple endocrine neoplasia type $2 \mathrm{~A}$ Nature 363: 458-460.

12. Sorbi S, Nacmias B, Forleo P, Piacentini S, Sherrington R, et al. (1995) Missense mutation of $\mathrm{S} 182$ gene in Italian families with early-onset Alzheimer's disease. Lancet 346: 439-440.

13. Levy-Lahad E, Wasco W, Poorkaj P, Romano DM, Oshima J, et al. (1995) Candidate gene for the chromosome 1 familial Alzheimer's disease locus. Science 269: 973-977.

14. Levy-Lahad E, Wijsman EM, Nemens E, Anderson L, Goddard KA, et al. (1995) A familial Alzheimer's disease locus on chromosome 1. Science 269: 970-973.

15. Tanzi RE, Bertram L (2005) Twenty years of the Alzheimer's disease amyloid hypothesis: a genetic perspective. Cell 120: 545-555

16. De Strooper B (2003) Aph-1, Pen-2, and Nicastrin with Presenilin generate an active gamma-Secretase complex. Neuron 38: 9-12. 
17. Li D, Parks SB, Kushner JD, Nauman D, Burgess D, et al. (2006) Mutations of presenilin genes in dilated cardiomyopathy and heart failure. Am J Hum Genet 79: 1030-1039.

18. Gianni D, Li A, Tesco G, McKay KM, Moore J, et al. (2010) Protein aggregates and novel presenilin gene variants in idiopathic dilated cardiomyopathy. Circulation 121: 1216-1226.

19. Wang B, Yang W, Wen W, Sun J, Su B, et al. (2010) Gamma-secretase gene mutations in familial acne inversa. Science 330: 1065.

20. Bertram L, Tanzi RE (2008) Thirty years of Alzheimer's disease genetics: the implications of systematic meta-analyses. Nat Rev Neurosci 9: 768-778.

21. Scheuner D, Eckman C, Jensen M, Song X, Citron M, et al. (1996) Secreted amyloid beta-protein similar to that in the senile plaques of Alzheimer's disease is increased in vivo by the presenilin 1 and 2 and APP mutations linked to familial Alzheimer's disease. Nat Med 2: 864-870.

22. Tanzi RE (1999) A genetic dichotomy model for the inheritance of Alzheimer's disease and common age-related disorders. J Clin Invest 104: 1175-1179.

23. Jayadev S, Leverenz JB, Steinbart E, Stahl J, Klunk W, et al. (2010) Alzheimer's disease phenotypes and genotypes associated with mutations in presenilin 2. Brain 133: 1143-1154.

24. Chan SL, Mayne M, Holden CP, Geiger JD, Mattson MP (2000) Presenilin-1 mutations increase levels of ryanodine receptors and calcium release in $\mathrm{PC} 12$ cells and cortical neurons. J Biol Chem 275: 18195-18200.

25. Chan SL, Culmsee C, Haughey N, Klapper W, Mattson MP (2002) Presenilin-1 mutations sensitize neurons to DNA damage-induced death by a mechanism involving perturbed calcium homeostasis and activation of calpains and caspase-12. Neurobiol Dis 11: 2-19.

26. De Strooper B (2007) Loss-of-function presenilin mutations in Alzheimer disease. Talking Point on the role of presenilin mutations in Alzheimer disease. EMBO Rep 8: 141-146.

27. Lu Y, Lv Y, Ye Y, Wang Y, Hong Y, et al. (2007) A role for presenilin in post-stress regulation: effects of presenilin mutations on $\mathrm{Ca} 2+$ currents in Drosophila. FASEB J 21: 2368-2378.

28. Dineley KT, Xia X, Bui D, Sweatt JD, Zheng H (2002) Accelerated plaque accumulation, associative learning deficits, and up-regulation of alpha 7 nicotinic receptor protein in transgenic mice co-expressing mutant human presenilin 1 and amyloid precursor proteins. J Biol Chem 277: 22768-22780.

29. Saura CA, Choi SY, Beglopoulos V, Malkani S, Zhang D, et al. (2004) Loss of presenilin function causes impairments of memory and synaptic plasticity followed by age-dependent neurodegeneration. Neuron 42: 23-36.

30. Grogan M, Redfield MM, Bailey KR, Reeder GS, Gersh BJ, et al. (1995) Long- term outcome of patients with biopsy-proved myocarditis: comparison with idiopathic dilated cardiomyopathy. J Am Coll Cardiol 26: 80-84.

31. Burkett EL, Hershberger RE (2005) Clinical and genetic issues in familia dilated cardiomyopathy. J Am Coll Cardiol 45: 969-981.

32. Schönberger J, Seidman CE (2001) Many roads lead to a broken heart: the genetics of dilated cardiomyopathy. Am J Hum Genet 69: 249-260.

33. Olson EN (2004) A decade of discoveries in cardiac biology. Nat Med 10: 467 474

34. Thinakaran G, Borchelt DR, Lee MK, Slunt HH, Spitzer L, et al. (1996) Endoproteolysis of presenilin 1 and accumulation of processed derivatives in vivo. Neuron 17: 181-190.

35. Mah AL, Perry G, Smith MA, Monteiro MJ (2000) Identification of ubiquilin, a novel presenilin interactor that increases presenilin protein accumulation. $J$ Cel Biol 151: 847-862.

36. Li A, Xie Z, Dong Y, McKay KM, McKee ML, et al (2007) Isolation and characterization of the Drosophila ubiquilin ortholog dUbqln: in vivo interaction with early-onset Alzheimer disease genes. Hum Mol Genet 16: 2626-2639.

37. Schellenberg GD (1995) Genetic dissection of Alzheimer disease, a heterogeneous disorder. Proc Natl Acad Sci U S A 92: 8552-8559.

38. Cruts M, Hendriks L, Van Broeckhoven C (1996) The presenilin genes: a new gene family involved in Alzheimer disease pathology. Hum Mol Genet 5: 1449 1455.

39. Lendon CL, Ashall F, Goate AM (1997) Exploring the etiology of Alzheimer disease using molecular genetics. JAMA 277: 825-831.

40. To MD, Gokgoz N, Doyle TG, Donoviel DB, Knight JA, et al. (2006) Functiona characterization of novel presenilin-2 variants identified in human breast cancers. Oncogene 25: 3557-3564.

41. Donoviel DB, Hadjantonakis AK, Ikeda M, Zheng H, Hyslop PS, et al. (1999) Mice lacking both presenilin genes exhibit early embryonic patterning defects. Genes Dev 13: 2801-2810.

42. Nakajima M, Moriizumi E, Koseki H, Shirasawa T (2004) Presenilin 1 is essential for cardiac morphogenesis. Dev Dyn 230: 795-799.

43. Li A, Zhou C, Moore J, Zhang P, Tsai TH, et al. (2011) Changes in the expression of the Alzheimer's disease-associated presenilin gene in drosophila heart leads to cardiac dysfunction. Curr Alzheimer Res 8: 313-322.

44. Lasko LA, Post C, Kathju S (2008) Hidradenitis suppurativa: a disease of apocrine gland physiology. JAAPA 21: 23-25

45. Gao M, Wang PG, Cui Y, Yang S, Zhang YH, et al. (2006) Inversa acne (hidradenitis suppurativa): a case report and identification of the locus at chromosome 1p21.1-1q25.3. J Invest Dermatol 126: 1302-1306. 